\title{
Reversible and irreversible dynamics of a qubit interacting with a small environment
}

\author{
Giuliano Benenti ${ }^{1}$ and G. Massimo Palma ${ }^{2}$ \\ ${ }^{1}$ CNISM, CNR-INFM and Center for Nonlinear and Complex Systems, Università degli Studi dell'Insubria, via Valleggio 11, I-22100 \\ Como, Italy and Istituto Nazionale di Fisica Nucleare, Sezione di Milano, via Celoria 16, I-20133 Milano, Italy \\ ${ }^{2}$ NEST_CNR (INFM) and Dipartimento di Scienze Fisiche ed Astronomiche, Università di Palermo, \\ via Archirafi 36, I-90123 Palermo, Italy \\ (Received 27 November 2006; published 21 May 2007)
}

\begin{abstract}
We analyze the dynamics of a system qubit interacting by means of a sequence of pairwise collisions with an environment consisting of just two qubits. We show that the density operator of the qubits approaches a common time-averaged equilibrium state, characterized by large fluctuations, only for a random sequence of collisions. For a regular sequence of collisions the qubit states of the system and of the reservoir undergo instantaneous periodic oscillations and do not relax to a common state. Furthermore we show that pure bipartite entanglement is developed only when at least two qubits are initially in the same pure state while otherwise also genuine multipartite entanglement builds up.
\end{abstract}

DOI: 10.1103/PhysRevA.75.052110

PACS number(s): 03.65.Yz, 03.67.Mn, 03.67.Pp

\section{INTRODUCTION}

Quantum information is providing new perspectives and is opening new issues in the analysis of the dynamics of open quantum systems. For instance in [1] the loss of coherence of a system of qubits has been shown to be linked to the irreversible flow of information between the system and the bath due to a build up of entanglement between the two. Such model has been extended to the case in which couplings between the bath degrees of freedom are present [2-5]. In particular it was shown that, due to the monogamy of entanglement [6], the presence of intrabath entanglement can inhibit decoherence processes. The irreversible dissipation of a qubit has been analyzed in [7-9] in terms of an exchange of information by means of repeated collisions between a single qubit and a reservoir of an arbitrarily large number $N$ of identical independent qubits all prepared in the same state. In most of the existing literature an irreversible dynamics is obtained under the assumption that the reservoir has a large number of degrees of freedom. For instance, in the derivation of master equations the Born-Markov approximation is justified on the basis of a weak coupling with a basically unaffected large reservoir. On the other hand, the dynamics of a system interacting with a small environment can have rich new features ranging from memory effects, Poincaré recurrences, etc. Our work is somehow in the same spirit of the works by Mahler and co-workers [10], in which the interaction of various quantum systems with finite environments, like finite quantum networks or quantum Turing machines, has been analyzed with particular focus on the onset of quantum chaos. In the present paper we will address the problem whether, and under which conditions, an irreversible dynamics can appear when a system interacts with very small reservoirs, a situation often encountered in the physics of mesoscopic systems [11] and of quantum information processing $[12,13]$ and of growing interest. To this goal we will use a repeated collision model to analyze the time evolution of a qubit interacting with the smallest nontrivial reservoir consisting of just two qubits. In spite of the apparent simplicity of the model, the system dynamics shows interest- ing new features. Due to the small size of the environment, the reduced dynamics of the system is characterized by large fluctuations. However an irreversible dynamics is retrieved when the system dynamics is averaged over a sufficiently large number of collisions (as we will discuss later this is different from the usual time coarse grained introduced in the derivation of a master equation). We will show that a timeaveraged equilibrium state is reached only for a random sequence order of collisions. This is not a priori an obvious result. It is important to stress that the reduced dynamics of the system qubit cannot, in this case, be described in terms of a Markovian master equation, as we will show below.

\section{THE MODEL}

To set the scenario and to illustrate the approach of our work let us review the repeated collision model. Consider a set of $N+1$ qubits, the first of which is the system qubit and the remaining $N$ are the reservoir. The interaction between system and environment is due to pairwise collisions between the system and a singe reservoir qubit. Each collision is described in terms of a unitary operator $U_{i}$. After $t$ collisions the overall state of the system plus reservoir is

$$
\varrho_{S E}^{(t)}=U_{i_{t}} \cdots U_{i_{2}} U_{i_{1}} \varrho_{S E}^{(0)} U_{i_{1}}^{\dagger} U_{i_{2}}^{\dagger} \cdots U_{i_{t}}^{\dagger},
$$

where $\varrho_{S E}$ is the total density operator and the sequence of labels $i_{1} \cdots i_{t}$ specify the order with which the environment qubits collide with the system one. In general each collision modifies the entanglement between the system and the environment qubits, in particular initially separable qubits will become entangled. Such model was considered to analyze the processes of thermalization [7] and of homogenization $[8,9]$. These were shown to be achieved when the collisions are described by the partial swap operator

$$
U_{i}=\cos \eta \mathbb{1}+i \sin \eta S_{i},
$$

where $S_{i}$ is the swap operator between the system qubit and the $i$ th environment qubit defined by the relation $S_{i}|\psi\rangle$ $\otimes|\phi\rangle=|\phi\rangle \otimes|\psi\rangle$ (here $|\psi\rangle \otimes|\phi\rangle$ is an arbitrary product of 

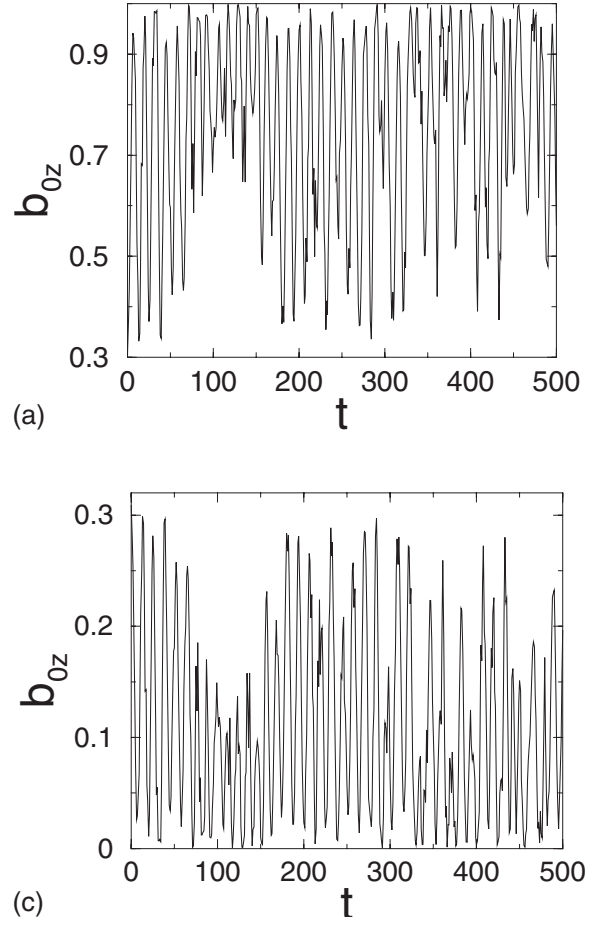
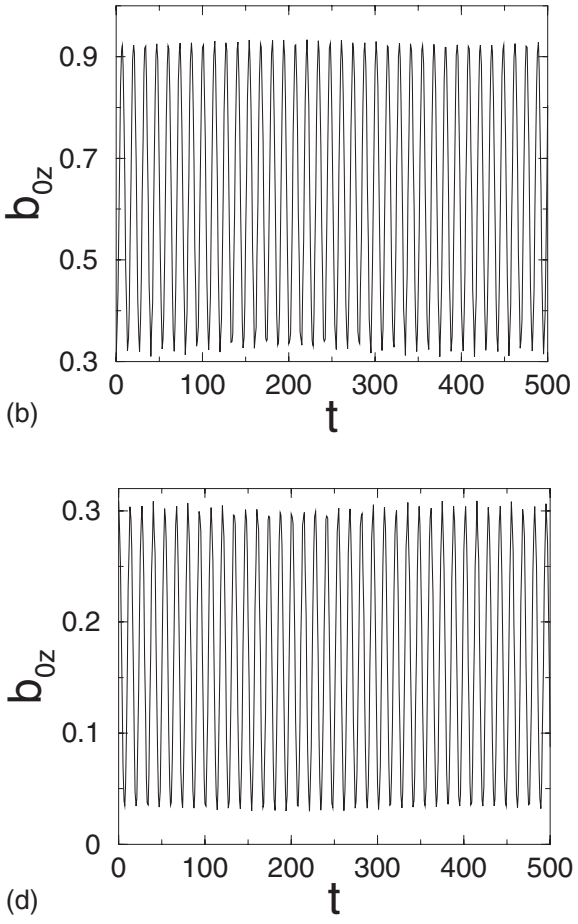

FIG. 1. Time evolution of the $z$ component of the system qubit Bloch vector $\vec{b}_{0}$, for a random (left) or an ordered (right) sequence of collisions. The initial condition for the environment is the separable state $|00\rangle$ (above) or the Bell state $\frac{1}{\sqrt{2}}(|00\rangle+|11\rangle)$ (below). Parameter values: $\eta=\frac{1}{10} \pi$, $\theta=\frac{2}{5} \pi$. single-qubit pure states). The environment was assumed to consist of a set of $N$ qubits all initially prepared in the (generally mixed) state $\xi$. For instance in [7] $\xi$ is a thermal state of a single spin. The initial density operator was therefore $\varrho_{S E}^{(0)}=\varrho_{S}^{(0)} \otimes \xi^{\otimes N}$. The number $N$ of environment qubits was assumed to be infinite in order to neglect repeated collisions between the system qubit and the same environment qubit. Under these assumption the system qubit was shown to relax to thermal equilibrium. More in general, such model describes a homogenization process in which the qubit system approaches state $\xi[8]$. Such relaxation mechanism can be understood easily if one notes that this models contains all the ingredients used in standard derivations of a master equation: the system interacts with an environment which rapidly "forgets" the effects of the coupling with the system and returns to its stationary state.

Here we will look at the model from an entirely different viewpoint analyzing the system dynamics in the opposite limit of the smallest nontrivial environment, namely the limit $N=2$. The reason for this choice is twofold. On the one hand, although thermalization or homogenization are clearly not possible for such small environment we would like to know if, and under which conditions, the system and the environment reach some form of equilibrium. Furthermore, since the entanglement dynamics among the three qubits can be followed and characterized in detail, it is possible to analyze under which conditions bipartite entanglement and genuine tripartite entanglement builds up.

\section{SMALL ENVIRONMENT}

The major new feature of the small reservoir limit is clearly the fact that the system qubit collides repeatedly with the same environment qubits. In our case Eq. (1) reduces to

$$
\varrho_{012}^{(t)}=U_{i_{t}} \cdots U_{i_{2}} U_{i_{1}} \varrho_{012}^{(0)} U_{i_{1}}^{\dagger} U_{i_{2}}^{\dagger} \cdots U_{i_{t}}^{\dagger},
$$

where the label 0 refers to the system qubit while 1,2 refer to the environment qubits. We will not restrict ourselves to the case in which $\varrho_{012}^{(0)}=\varrho_{0} \otimes \varrho_{1} \otimes \varrho_{2}$ but we will consider also the case in which some entanglement is initially present between the environment qubits. The order with which the $t$ collisions take place is specified by the string of indices $i_{1} \cdots i_{t}$. We have considered two limiting cases: a completely random sequence, corresponding to the situation in which the system qubit collides with equal probability with each of the environment qubits, and the regular periodic sequence $1212 \cdots 12 \cdots$, corresponding to the situation in which the system qubits collides alternatively with the two environment qubits.

\section{A. Approach to equilibrium}

In order to characterize the approach to equilibrium we will make use of an important feature of our system: the total Bloch vector, defined as $\overrightarrow{\mathcal{B}}=\sum_{i=0}^{2} \vec{b}_{i}$, where $\vec{b}_{i}$ is $i$ th qubit Bloch vector, is a constant of motion. If the state of both system and environment qubits approach the same equilibrium reduced density operator, after a sufficiently long number of collisions we must have $\vec{b}_{i} \rightarrow \overrightarrow{\mathcal{B}} / 3$. In Fig. 1 we show the time evolution of the $z$ component of the system qubit Bloch vector $\vec{b}_{0}$, for a random and an ordered sequence of collisions. A similar behavior is shown by the $x$ and $y$ components (data not shown). The system qubit is assumed to be in the state $\cos \left(\frac{\theta}{2}\right)|0\rangle+\sin \left(\frac{\theta}{2}\right)|1\rangle$ while the environment is assumed to be initially in the state $|00\rangle$ or in the Bell state $\frac{1}{\sqrt{2}}(|00\rangle+|11\rangle)$. Such choice has been made to illustrate the new features of the dynamics for two different instances of 

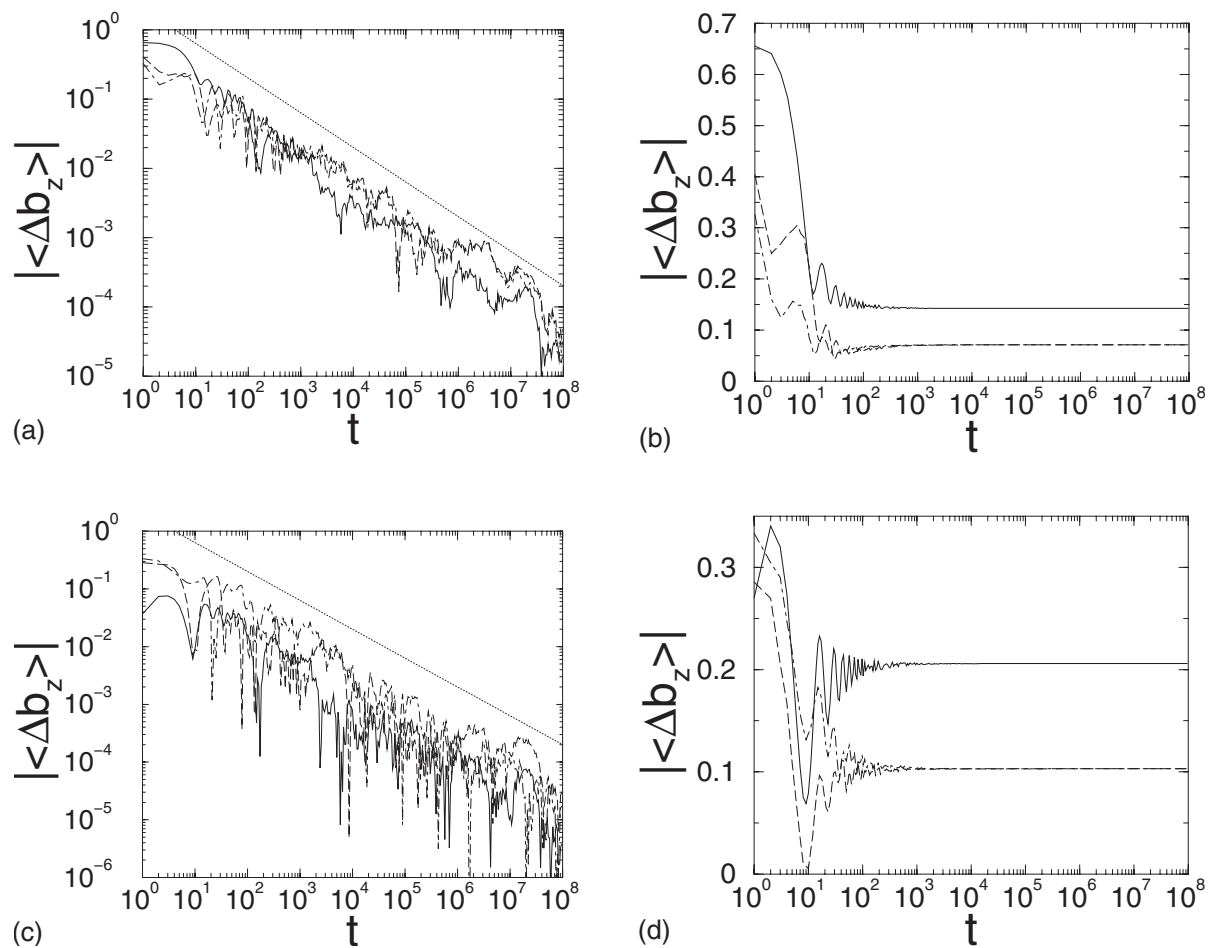

FIG. 2. Length $\left|\left\langle\Delta b_{i z}\right\rangle\right|$ of the $z$ component of the vector $\left\langle\Delta \vec{b}_{i}\right\rangle$, for the system qubit $(i=0$, solid curves) and the environment qubits $(i=1,2$, dashed and dotteddashed curves, respectively). The straight lines show a decay $\propto t^{-1 / 2}$. Same initial conditions and parameter values as in Fig. 1. environment in a pure state with an equal reduced density operator for the two environment qubits and with different bipartite initial entanglement.

The figures clearly show that while for the ordered sequence of collisions the $z$ component of the Bloch vector exhibits periodic oscillations, strong and rapid fluctuations appear for a random sequence of collisions, due to the extremely small size of our reservoir. This is very different from what happens in the large reservoir limit, where, for sufficiently weak collisions, i.e., for $\eta$ small enough, the environment qubits are hardly modified by the interaction with the system, while, after a sufficiently large number of collisions the density operator of the system qubit becomes monotonically arbitrarily close to $\xi[7,8]$.

In our system a steady state is however reached if one looks at time-averaged dynamics. In order to explore the emergence of a time-averaged equilibrium state, we have evaluated the components of the vector

$$
\left\langle\Delta \vec{b}_{i}\right\rangle(t)=\frac{1}{t+1} \sum_{t^{\prime}=0}^{t}\left(\vec{b}_{i}\left(t^{\prime}\right)-\frac{\overrightarrow{\mathcal{B}}}{3}\right)=\left\langle\vec{b}_{i}\right\rangle(t)-\frac{\overrightarrow{\mathcal{B}}}{3},
$$

where $\langle A\rangle(t)$ denotes the time average of the quantity $A$ from time 0 to time $t$ and $i$ labels the qubits. In Fig. 2 the length $\left|\left\langle\Delta b_{i z}\right\rangle\right|$ of the $z$ component of the vector $\left\langle\Delta \vec{b}_{i}\right\rangle$, for the system and the environment qubits is plotted, for the same initial conditions and parameter values as in Fig. 1. It is important to note that in the time-averaged dynamics one loses knowledge of the exact sequence of collisions. If the same time average were done in the homogenization process described in [8], all sequence of collisions would give origin to the same irreversible dynamics. This is not at all the case in the small reservoir limit. Our results suggest that the ap- proach to a time-averaged equilibrium state depends on how random-i.e., how compressible in the Kolmogorov sense [14] - the string identifying the sequence of collisions is. When a regular pattern exists in the collision sequence then the time-averaged dynamics of the system and the reservoir qubits settle on different values. Note incidentally that while in the large $N$ limit, thanks to the fact that the system qubit collides with "fresh" reservoir qubits, the system dynamics can be analyzed in terms of completely positive (CP) maps forming a semigroup leading to an effective Lindblad time evolution [9], this is not possible in our case, since, due to the repeated collisions among the same qubits, the state of reservoir qubits involved in the collision changes each time and an irreversible dynamics is obtained only after time average. We emphasize that our time average is different from the typical coarse graining introduced in the derivation of a Markovian master equation. Such coarse graining amounts to looking at the system dynamics over a time scale longer than the time needed for the reservoir to reset to its steady state. In the repeated collision model with an infinite reservoir model the environment sets to its equilibrium value after each individual collision as the system collides with different fresh reservoir qubits.

The straight lines in the left-hand plots of Fig. 2 show a decay $\Delta b_{i z} \propto t^{-1 / 2}$ for a random sequence of collisions. This implies that the cumulative sum obtained by adding the vectors $\Delta \vec{b}_{i}\left(t^{\prime}\right)=\vec{b}_{i}\left(t^{\prime}\right)-\frac{\overrightarrow{\mathcal{B}}}{3}$ from time $t^{\prime}=0$ to time $t^{\prime}=t$ grows $\propto t^{1 / 2}$. Such diffusive growth is the same as for Brownian motion or for coin-tossing sequences and suggests that, given $t_{1}$ and $t_{2}$, the vectors $\Delta \vec{b}_{i}\left(t_{1}\right)$ and $\Delta \vec{b}_{i}\left(t_{2}\right)$ are in practice uncorrelated, provided $\left|t_{1}-t_{2}\right|$ is sufficiently large.

Further insight into the approach to equilibrium is gained by evaluating the self-correlation defined as 

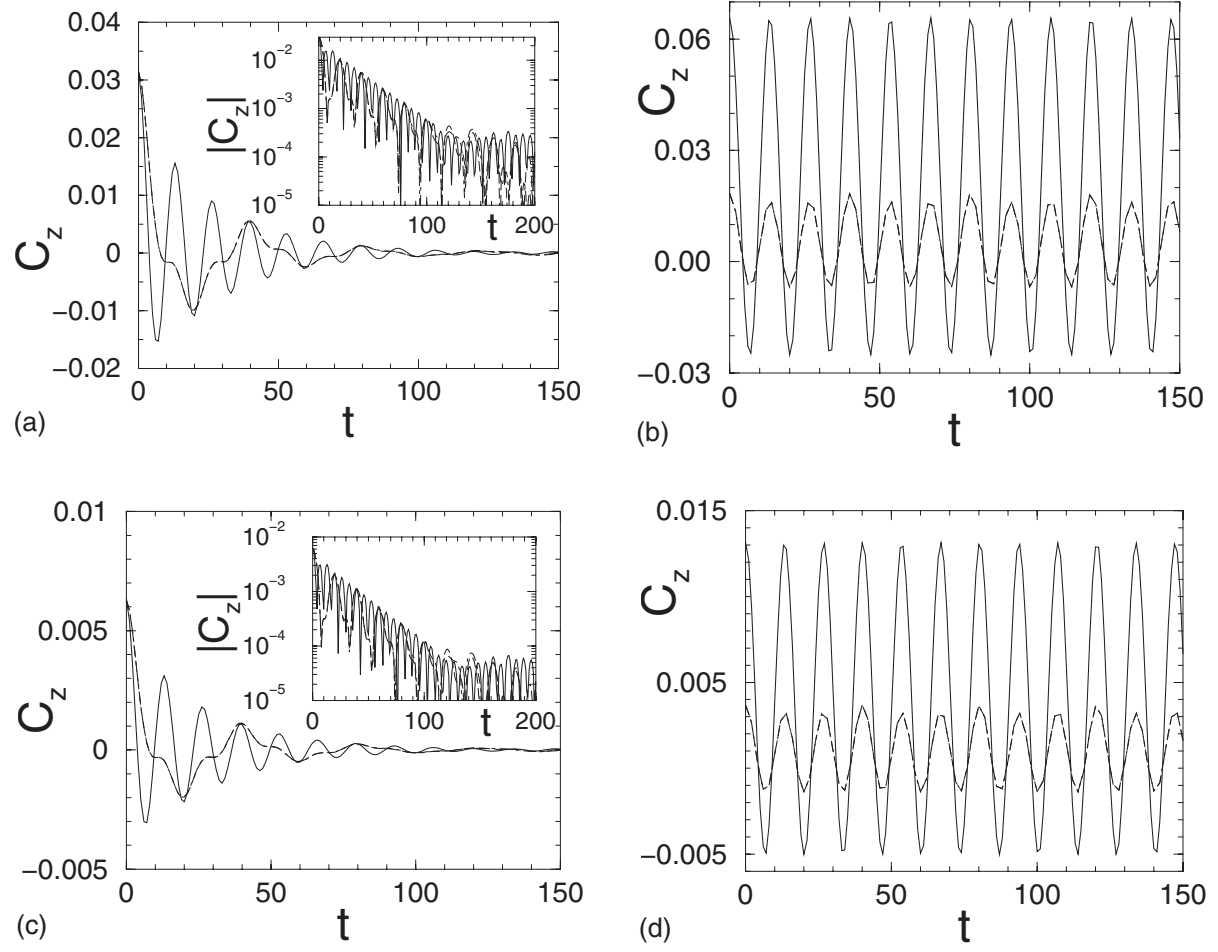

FIG. 3. Decay of the correlation function $C_{i z}$ (left-hand plots, corresponding to a random sequence of collisions) versus oscillations of the same correlation function in the case of a regular sequence of collisions (right-hand plots). Same meaning of the curves as in Fig. 2, initial conditions and parameter values as in Fig. 1. Insets: $\left|C_{i z}\right|$ vs time (semilogarithmic plot).

$$
\vec{C}_{i k}(t)=\lim _{T \rightarrow \infty} \frac{1}{T+1} \sum_{t^{\prime}=0}^{T} \Delta b_{i k}\left(t^{\prime}\right) \Delta b_{i k}\left(t^{\prime}+t\right),
$$

where $k=x, y, z$. In Fig. 3, we show the decay of the correlation function $C_{i z}$ (left-hand plots, corresponding to random sequence of collisions) versus the oscillations of the same correlation function in the case of a regular sequence of collisions (right-hand plots). The insets show the exponential decay of $\left|C_{i z}\right|$ when the sequence of collisions is random. Note that $\left|C_{i z}\right|$ eventually oscillates around a value $\propto T^{-1 / 2}$ due to the finite sequence of data considered in computing the correlation function (in these plots, $T=10^{6}$ ). This is again compatible with normal diffusive dynamics.

\section{B. Entanglement dynamics}

Interesting features of our model appear when the time evolution of bipartite and multipartite entanglement is analyzed. To this end let us first introduce the tangle as a measure of bipartite entanglement. Given the density operator $\rho_{a b}$ of a bipartite system of two qubits, the tangle $\tau_{a \mid b}$ is defined as

$$
\tau_{a \mid b}(\rho)=\left[\max \left\{0, \alpha_{1}-\alpha_{2}-\alpha_{3}-\alpha_{4}\right\}\right]^{2},
$$

where $\left\{\alpha_{i}\right\}(i=1, \ldots, 4)$ are the square roots of the eigenvalues (in nonincreasing order) of the non-Hermitian operator $\bar{\rho}=\rho\left(\sigma_{y} \otimes \sigma_{y}\right) \rho^{*}\left(\sigma_{y} \otimes \sigma_{y}\right), \sigma_{y}$ is the $y$-Pauli operator and $\rho^{*}$ is the complex conjugate of $\rho$, in the eigenbasis of $\sigma_{z} \otimes \sigma_{z}$ operator. In our model the tangle $\tau_{j \mid k}$ can be used to quantify the entanglement between the pair of qubits $i, j$ for an arbitrary reduced density operator $\rho_{i j}$. Furthermore, when the overall state of the system is pure, the amount of entanglement between qubit $j$ and all the remaining can be quantified by the tangle $\tau_{j \mid \text { rest }}=4 \operatorname{det} \rho_{j}$. We have numerically computed the tangles $\tau_{0 \mid 1}, \tau_{0 \mid 2}$, and $\tau_{1 \mid 2}$ of the two-qubit reduced density matrices and the three-tangle $\tau_{i|j| k}=\tau_{i \mid j k}-\tau_{i \mid j}-\tau_{i \mid k}$, where $i, j, k$ can take values $0,1,2$ and where the tangle $\tau_{i \mid j k}$ measures the entanglement between the $i$ th qubit and the rest of the system, i.e., qubits $j, k$. The three-tangle $\tau_{0|1| 2}$ is a measure of the purely tripartite entanglement and is invariant under permutations of the three qubits [6]. Our numerical results of Fig. 4 show that, for a random sequence of collisions, the time-averaged tangles $\left\langle\tau_{0 \mid 1}\right\rangle,\left\langle\tau_{0 \mid 2}\right\rangle$, and $\left\langle\tau_{1 \mid 2}\right\rangle$ saturate to the same limiting value. This confirms once more the approach to statistical equilibrium for our system. Moreover, we can see from Fig. 4 that genuine multipartite entanglement, i.e., $\left\langle\tau_{0|1| 2}\right\rangle \neq 0$, is created when the two environment qubits are initially prepared in a Bell state, but not if they are described by the same separable initial state. More generally, purely bipartite entanglement builds up among the qubits when at least two qubits (either both environment qubits or system and one environment qubit) are in the same state and the initial state is a pure separable state. Indeed, if the initial state $\left|\psi_{S E}^{(0)}\right\rangle$ is $|\psi \phi \phi\rangle$, or $|\phi \psi \phi\rangle$, or $|\phi \phi \psi\rangle$, then after $t$ collisions we obtain $\left|\psi_{S E}^{(t)}\right\rangle=\alpha(t)|\psi \phi \phi\rangle+\beta(t)|\phi \psi \phi\rangle+\gamma(t)|\phi \phi \psi\rangle$, where the coefficients $\alpha, \beta, \gamma$ are determined by the collision sequence. Using local unitary transformations only, i.e., with a tensor product of single-qubit unitary transformations, we can map this state into $a(t)|000\rangle+b(t)|100\rangle+c(t)|010\rangle$ $+d(t)|001\rangle$, where $a, b, c, d \geqslant 0$ and $a^{2}+b^{2}+c^{2}+d^{2}=1$. This latter is the standard form for the states of the $W$ class [15], which are characterized by purely bipartite entanglement. However, for general initial conditions, genuine multipartite entanglement builds up, as is the case when the two environment qubits are initially in a Bell state. We point out however that the initial presence of bipartite entanglement in the res- 

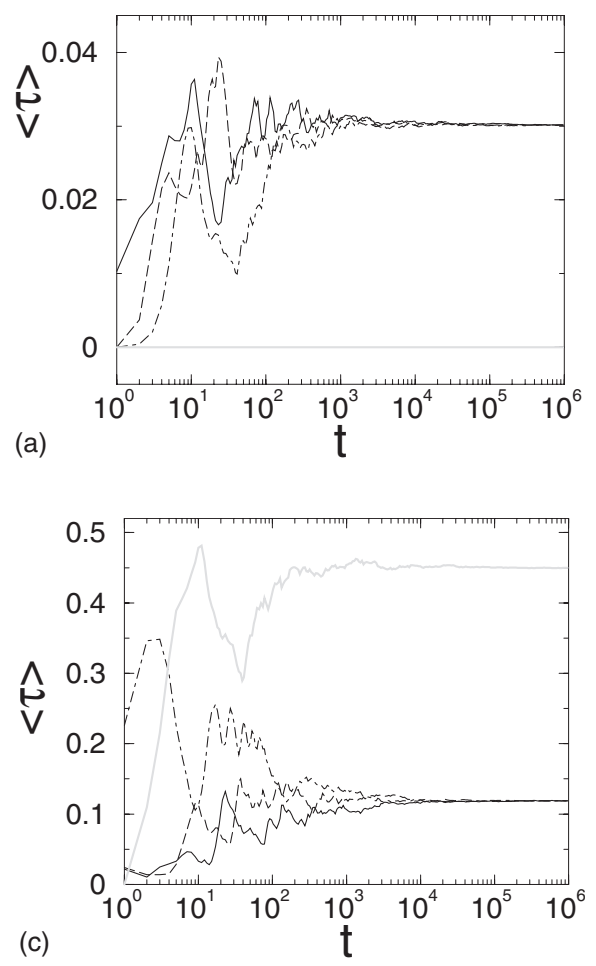
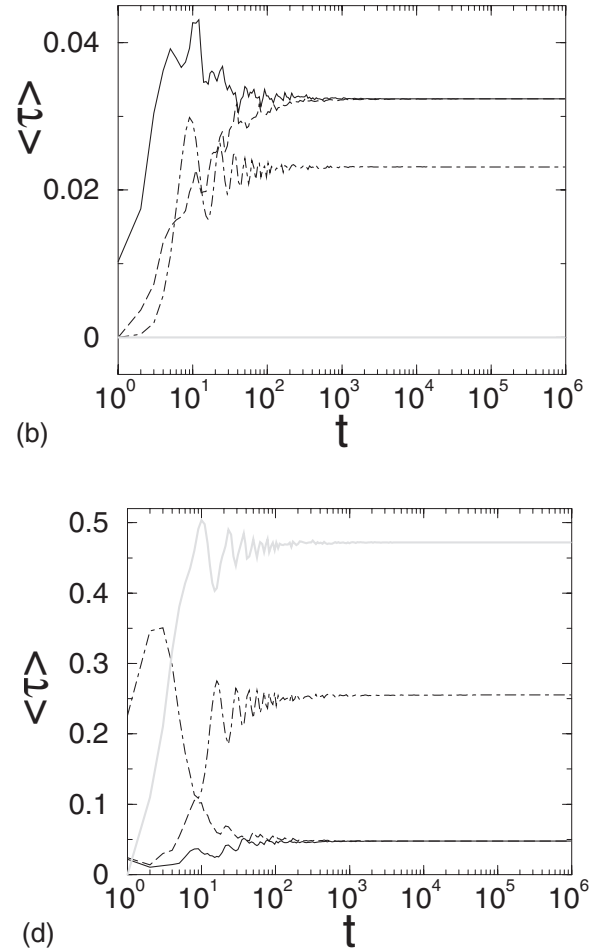

FIG. 4. Time-averaged tangles $\left\langle\tau_{0 \mid 1}\right\rangle$ (solid curves), $\left\langle\tau_{0 \mid 2}\right\rangle$ (dashed curves), $\left\langle\tau_{1 \mid 2}\right\rangle \quad$ (dotted-dashed curves), and $\left\langle\tau_{0|1| 2}\right\rangle$ (gray curves). Same initial conditions and parameter values as in Fig. 1. ervoir is not a necessary condition for the appearance of multipartite entanglement. Indeed, as should be clear from the symmetry argument outlined above, a nonzero timeaveraged three tangle $\left\langle\tau_{0|1| 2}\right\rangle \neq 0$ builds up as long as the three qubits are initially in different pure states. On the other hand, if the initial state of the three qubits is of the Greenberger-Horne-Zeilinger (GHZ) family the multipartite entanglement would remain purely tripartite. This of course is due to the fact that the partial swap operator does not change the overall number of $|0\rangle$ and $|1\rangle$ states.

\section{CONCLUSIONS}

In summary, we have shown that relaxation (in time average) to statistical equilibrium is possible for a system of just three qubits undergoing purely unitary evolution, provided that randomness is present in the sequence of pairwise collisions. We point out that the results of this paper can be easily extended to the case in which the environment consists of a finite number $N$ of qubits. In particular, a time-averaged equilibrium state characterized by purely bipartite entanglement is approached if the initial state is separable and $N$ qubits are in the same state (either all $N$ environment qubits or the system and $N-1$ environment qubits). In this case, the $N+1$-qubit state evolves in a subspace of states of the $W$ class (spanned by the "ground state" $|0 \cdots 0\rangle$ and the "singlequbit excitations" $|10 \cdots 0\rangle,|010 \cdots 0\rangle, \ldots,|0 \cdots 01\rangle)$, whose dimension is $N+1$, much smaller than the overall dimension $2^{N+1}$ of the Hilbert space. Therefore, the equilibrium state can be attained even though genuine multipartite entanglement is not developed. This implies that in a small, unitarily evolving quantum system statistical relaxation is possible even though the dynamics is restricted to a subspace whose states are characterized by purely bipartite entanglement. Since randomly chosen pure states in a many-qubit Hilbert space typically exhibit large multipartite entanglement [16], it follows that relaxation to equilibrium is possible without exploiting the full complexity of the Hilbert space.

\section{ACKNOWLEDGMENTS}

One of the authors (G.B.) acknowledges support from the PRIN 2005 "Quantum computation with trapped particle arrays, neutral and charged." One of the authors (G.M.P.) acknowledges support from the PRIN 2006 "Quantum noise in mesoscopic systems." The authors acknowledge useful discussion with V. Scarani and V. Bužek.
[1] G. M. Palma, K.-A. Suominen, and A. K. Ekert, Proc. R. Soc. London, Ser. A 452, 567 (1996).

[2] L. Tessieri and J. Wilkie, J. Phys. A 36, 12305 (2003).

[3] S. Paganelli, F. de Pasquale, and S. M. Giampaolo, Phys. Rev. A 66, 052317 (2002)
[4] M. Lucamarini, S. Paganelli, and S. Mancini, Phys. Rev. A 69, 062308 (2004).

[5] C. M. Dawson, A. P. Hines, R. H. McKenzie, and G. J. Milburn, Phys. Rev. A 71, 052321 (2005).

[6] V. Coffman, J. Kundu, and W. K. Wootters, Phys. Rev. A 61, 
052306 (2000).

[7] V. Scarani, M. Ziman, P. Štelmachovič, N. Gisin, and V. Bužek, Phys. Rev. Lett. 88, 097905 (2002).

[8] M. Ziman, P. Štelmachovič, V. Bužek, M. Hillery, V. Scarani, and N. Gisin, Phys. Rev. A 65, 042105 (2002); M. Ziman, P. Štelmachovič, and V. Bužek, J. Opt. B: Quantum Semiclassical Opt. 5, S439 (2003).

[9] M. Ziman, P. Štelmachovič, and V. Bužek, Open Syst. Inf. Dyn. 12, 81 (2005); M. Ziman and V. Bužek, Phys. Rev. A 72, 022110 (2005).

[10] I. Kim and G. Mahler, Phys. Lett. A 263, 268 (1999); J. Mod. Opt. 47, 177 (2000).

[11] G. Ithier, E. Collin, P. Joyez, P. J. Meeson, D. Vion, D. Esteve,
F. Chiarello, A. Shnirman, Y. Makhlin, J. Schriefl, and G. Schön, Phys. Rev. B 72, 134519 (2005).

[12] M. M. Wolf and D. Pérez-García, Phys. Rev. A 75, 012303 (2007).

[13] D. Kretschmann and R. F. Werner, Phys. Rev. A 72, 062323 (2005).

[14] G. J. Chaitin, Information, Randomness and Incompleteness (World Scientific, Singapore, 1987).

[15] W. Dür, G. Vidal, and J. I. Cirac, Phys. Rev. A 62, 062314 (2000).

[16] P. Facchi, G. Florio, and S. Pascazio, Phys. Rev. A 74, 042331 (2006). 